\title{
Design and test of the optical fiber assemblies for the scalar magnetic field sensor aboard the JUICE mission
}

Irmgard Jernej, M. Faust, R. Lammegger, I. McKenzie, J. Kuhnhenn, et al.

Irmgard Jernej, M. Faust, R. Lammegger, I. McKenzie, J. Kuhnhenn, C. Knothe, S. O'Riorden, J. Barbero, P. Brown, V. Lelievre, M. Agú, A. Alessi, C. Amtmann, A. Betzler, M. Dougherty, M. Ellmeier, C. Hagen, A. Hauser, M. Hartig, A. Lamott, M. Leichtfried, W. Magnes, A. Mahapatra, S. Mariojouls, D. Monteiro, A. Pollinger, A. Salomon, U. Weinand, R. Wolf, "Design and test of the optical fiber assemblies for the scalar magnetic field sensor aboard the JUICE mission," Proc. SPIE 11852, International Conference on Space Optics - ICSO 2020, 1185264 (11 June 2021); doi: 10.1117/12.2600052

SPIE Event: International Conference on Space Optics - ICSO 2021, 2021, Online Only 


\section{International Conference on Space Optics-ICSO 2020}

Virtual Conference

30 March-2 April 2021

Edited by Bruno Cugny, Zoran Sodnik, and Nikos Karafolas
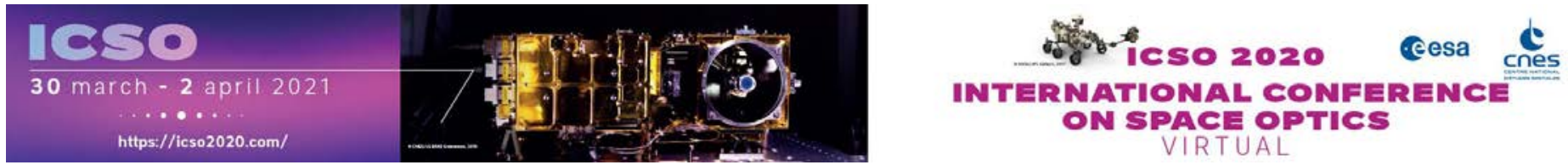

\section{Design and test of the optical fiber assemblies for the scalar magnetic field sensor aboard the JUICE mission}

\section{Cesa isoporecestings denes}




\title{
Design and Test of the Optical Fiber Assemblies for the Scalar Magnetic Field Sensor aboard the JUICE Mission
}

\author{
I. Jernej*a ${ }^{* a}$ M. Faust ${ }^{\mathrm{b}}$, R. Lammegger ${ }^{\mathrm{c}}$, I. McKenzie ${ }^{\mathrm{d}}$, J. Kuhnhenn ${ }^{\mathrm{e}}$, C. Knothe ${ }^{\mathrm{f}}$, S. O’Riorden ${ }^{\mathrm{g}}$, J.

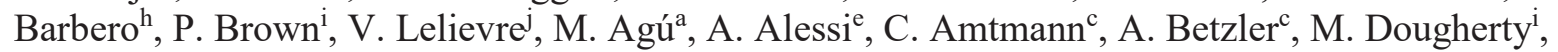

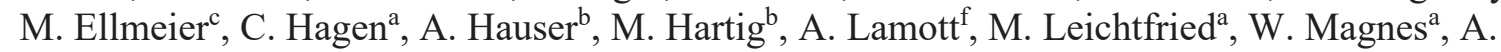 \\ Mahapatra $^{\mathrm{g}}$, S. Mariojouls ${ }^{\mathrm{j}}$, D. Monteiro ${ }^{\mathrm{d}}$, A. Pollinger ${ }^{\mathrm{a}}$, A. Salomon ${ }^{\mathrm{j}}, \mathrm{U}^{\mathrm{U}}$. Weinand ${ }^{\mathrm{e}}$ and R. Wolf ${ }^{\mathrm{e}}$

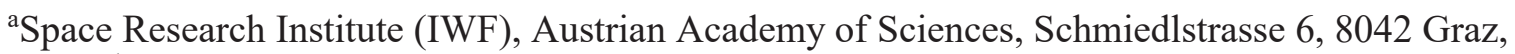 \\ Austria; ${ }^{b}$ Airbus Defense \& Space, Friedrichshafen, Claude-Dornier-Straße, 88090 Immenstaad am \\ Bodensee, Germany; ' Institute of Experimental Physics, Graz University of Technology, Petersgasse \\ 16, 8010 Graz, Austria; ${ }^{d}$ European Space Research and Technology Centre, Keplerlaan 1, 2201 AZ \\ Noordwijk, The Netherlands; ${ }^{\mathrm{E}}$ Fraunhofer INT, Appelsgarten 2, 53879 Euskirchen, Germany;

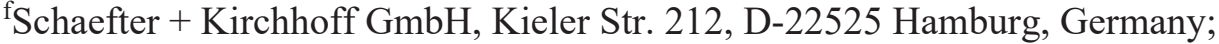 \\ ${ }^{g}$ Linden Photonics, Inc., 1 Park Drive, Unit 10, Westford, MA 01886, USA; ${ }^{\mathrm{h}}$ Alter Technology C/ \\ La Majada 328760 Tres Cantos - Madrid, Spain; ${ }^{i}$ Blackett Laboratory, Imperial College London, \\ London SW7 2BW, United Kingdom; ${ }^{\mathrm{j}}$ Airbus Defense \& Space, 31 Rue des Cosmonautes, 31400 \\ Toulouse, France
}

\begin{abstract}
A set of optical fiber assemblies has been developed and successfully qualified for its use on a European space science mission to the icy moons of Jupiter (Jupiter Icy Moons Explorer, JUICE), to be launched in 2022. The paper gives an overview of the design challenges, the test methods used for failure detection and screening of the optical fiber cable assemblies as well as the further testing performed in the frame of a lot acceptance qualification.
\end{abstract}

Keywords: Optic, fiber, jacket, connector, FC/APC, space, CTE, radiation

\section{INTRODUCTION}

The J-MAG instrument onboard ESA's JUICE mission to the icy moons of Jupiter is going to perform magnetic field measurements with the help of two vector (fluxgate) sensors and an additional optical scalar sensor (MAGSCA), which provides a reference for in-flight-calibration of the fluxgate sensors. The MAGSCA magnetometer uses a light source to establish Coherent Population Resonances (CPT) in a balanced configuration within the Hyperfine Structure (HFS) of the $87 \mathrm{Rb}$ isotope. Figure 1 shows a photo of the complete flight instrument. The optical sensor is located at the tip of a $10.5 \mathrm{~m}$ long boom whereas the light source (795 nm Vertical-Cavity Surface Emitting Laser; VCSEL) and the photodetector are part of the J-MAG instrument electronics located within the JUICE satellite. Two fiber-optical paths, with a routed length of nearly 20 meters each, are needed to transmit the laser light from the instrument electronics to the sensor and back. A $50 \mu \mathrm{m}$ graded-index multimode fiber was selected for the outbound optical transmission whereas the return path has been realized with a $400 \mu \mathrm{m}$ step index multimode fiber. Due to the JUICE mission's challenging environmental conditions in terms of particle radiation and temperature range as well as the need for a fully nonmagnetic design, a set of customized fiber-optical cable assemblies had to be designed and qualified.

*irmgard.jernej@oeaw.ac.at; phone +43 316 4120-565; https://www.oeaw.ac.at/en/iwf 


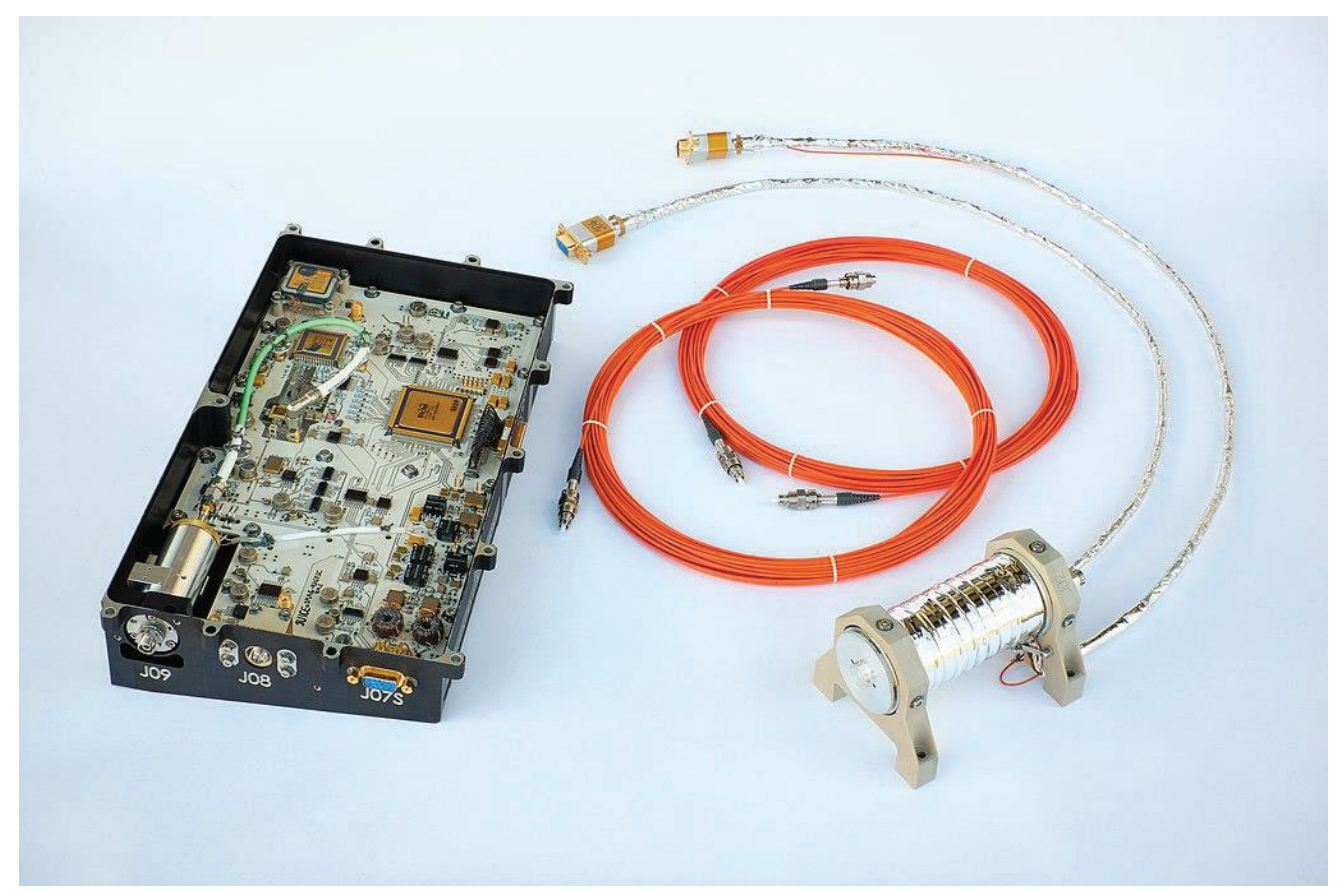

Figure 1. Flight unit of the JUICE J-MAG scalar magnetometer: electronics with laser unit (left), optical fiber assemblies (middle) and sensor (right) (Andreas Pollinger/IWF/OEAW, MAGSCA flight model, CC BY 4.0)

\section{OPTICAL FIBER ASSEMBLY DESIGN SPECIFICATION}

In the beginning of the JUICE project, the environmental specifications for the satellite's external optical harness along the magnetometer boom did include quite some margin for various uncertainties. These specifications have been updated later on after refined analysis and testing throughout the project. Table 1 shows the final specification of the optical fiber assemblies.

Table 1. Final specifications of the optical fiber assemblies for the JUICE J-MAG scalar magnetometer

\begin{tabular}{|c|c|}
\hline \multicolumn{2}{|l|}{ Optical fiber specification } \\
\hline Type of outgoing fiber (laser $\rightarrow$ sensor) & $50 \mu \mathrm{m}$ Graded Index Multimode Fiber \\
\hline Type of incoming fiber (sensor $\rightarrow$ photodiode) & $400 \mu \mathrm{m}$ Step Index Multimode Fiber \\
\hline Length of optical path per fiber type & $\sim 20 \mathrm{~m}$ \\
\hline \multicolumn{2}{|l|}{ Optical signal to be transmitted } \\
\hline Wave length of transmitted light in vacuum & $795 \mathrm{~nm}$ \\
\hline Optical power of transmitted light & $<250 \mu \mathrm{W}$ \\
\hline Bandwidth length product & $\geq 200 \mathrm{MHz}^{*} \mathrm{~km}$ \\
\hline \multicolumn{2}{|l|}{ Magnetic properties } \\
\hline Remanent flux density@2 cm distance & $<50 \mathrm{pT}$ (practically non-magnetic) \\
\hline
\end{tabular}




\begin{tabular}{|l|l|}
\hline \multicolumn{2}{|l|}{ Outgassing } \\
\hline Recovered Mass Loss (RML) or Total Mass Loss (TML) & $<1.0 \%$ \\
\hline Collected Volatile Condensable Material (CVCM) & $<0.1 \%$ \\
\hline Environmental conditions & $-190^{\circ} \mathrm{C}$ to $+120^{\circ} \mathrm{C}$ \\
\hline Optical fiber assembly temperature range & $-160^{\circ} \mathrm{C}$ to $+100^{\circ} \mathrm{C}$ \\
\hline Optical connectors temperature range & 10 Mrad \\
\hline Total Ionizing Dose (TID) & $\begin{array}{l}\text { Compatible with JUICE magnetometer } \\
\text { boom vibration and shock loads } \\
\text { (see Table 2) }\end{array}$ \\
\hline Vibration + Shock & \\
\hline
\end{tabular}

The environmental conditions were specified by Airbus Defense and Space [1], who carried the overall responsibility for the optical fiber assembly development for the J-MAG instrument on board of the JUICE mission. The optical specifications and magnetic properties were defined to the needs of the J-MAG scalar magnetometer by the instrument teams at the Institute of Experimental Physics of the Graz University of Technology (TUG), and the Space Research Institute (IWF) of the Austrian Academy of Sciences, Graz, Austria [2]. The whole design and qualification process was supported and guided by expertise from the European Space Agency (ESA).

\section{DESIGN CHALLENGES}

\subsection{Fiber and fiber coating}

High radiation tolerance at low temperatures was the main selection criterion for the optical fiber, availability was a second challenge. After literature studies (e.g., [3][14][23]) and discussions with several fiber manufacturers, pure silica fibers with fluorine doped core were selected for the graded index multimode fiber and high $\mathrm{OH}$-core silica fibers with fluorine doped cladding for the step index multimode fiber. As the upper temperature requirement at the time of optical fiber selection was $+200^{\circ} \mathrm{C}$, the choice of coating materials was limited as well. The usage of high temperature acrylate is restricted to $+150^{\circ} \mathrm{C}$, so only polyimide or metal coatings ( $\mathrm{Al}$ or $\mathrm{Au}$ ) remained. Silicone coating was excluded for its higher outgassing properties.

Finally, two $50 \mu \mathrm{m}$ fiber types and three $400 \mu \mathrm{m}$ fiber types from five different manufacturers were chosen for first Total Ionizing Dose (TID) radiation tests at cryogenic temperatures and different dose rates (see Section 6.2). From the results of this first test campaign, the two final flight fibers got selected: a $50 \mu \mathrm{m}$ fiber from Fibercore (GIMMSC(50/125)P) and a $400 \mu \mathrm{m}$ fiber from Molex/Polymicro (FVP400440480). Additional radiation tests were performed with the selected fibers to further evaluate the dose rate dependency of the Radiation Induced Attenuation (RIA) as well as photo bleaching effects. 


\subsection{Fiber jacket}

Meeting the above specifications is a major challenge for the production of a suitable fiber jacket. A jacket setup from GORE (as shown in Figure 2) - successfully deployed within a low Earth orbit mission [4][5] - was considered to be not suited for fiber optic assemblies in a thermal range as required for JUICE.

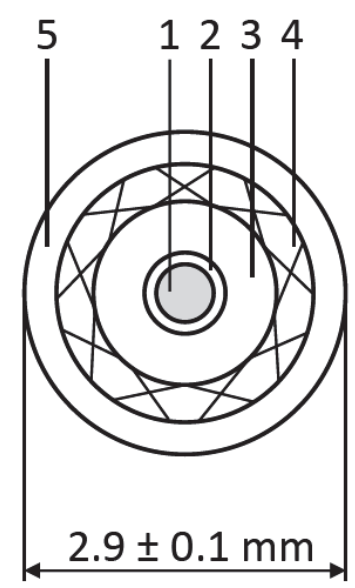

1. Optical Fiber: $400 \mu \mathrm{m} / 440 \mu \mathrm{m} / 625 \mu \mathrm{m}$ (core/cladding/coating)

2. Buffer: ePTFE

3. Sheath: PEEK

4. Braided Strain Relief: Kevlar

5. Jacket: FEP

Figure 2. The Gore GSC-13-84815-00 fiber optic multimode step-index cable [6] as used by IWF and TUG for the optical scalar magnetometer onboard a low Earth orbit space science mission [4]

Especially the large difference in the Coefficient of Thermal Expansion (CTE) between the optical fiber itself (made of fused silica; CTE is in the order of $0.5 \mathrm{ppm} / \mathrm{K}$ [7]) and the two stiffer jacket layers (made of Polyether Ether Ketone (PEEK) and Fluorinated Ethylene Propylene (FEP)) would have required to implement complex stress relief measures in order to avoid mechanical stress onto the fiber at the temperature extremes.

Therefore, a completely different fiber jacket setup has been chosen for JUICE. The jacket was developed based on an optical fiber cable design by Linden Photonics: The setup of the original Radiation Resistant, Non-kink Optical Cable (RAVNOC)[8] has been modified with the expertise of Linden Photonics and Schaefter+Kirchhoff, the manufacturer of the fiber connectors, to optimize the interfaces between fiber, jacket layers and connectors.

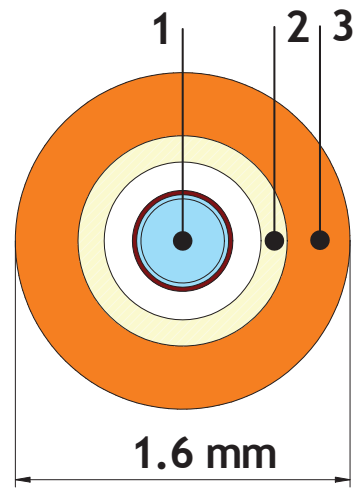

1. Optical Fiber: $400 \mu \mathrm{m} / 440 \mu \mathrm{m} / 480 \mu \mathrm{m}$ (core/cladding/coating)

2. Sheath / Strain Relief (Inner Jacket): LCP

3. Outer Jacket: FEP

Figure 3. Cross section of the $400 \mu \mathrm{m}$ optical fiber assembly developed for the JUICE mission [1]

A loose buffer design has been chosen. Figure 4 shows a cross section of the $400 \mu \mathrm{m}$ optical fiber assembly developed for JUICE. The sheath is made of Liquid Crystal Polymer (LCP), serving as both, sheath and strain relief at the same 
time. The advantage of this material is the low linear thermal expansion coefficient which matches better with the glass fiber than PEEK. FEP serves again as the outer jacket material. To reduce shrinking effects of the FEP [15][19], the jacket was cut close to the final fiber length and was thermally pre-conditioned for the first time prior to fiber insertion. The second and third thermal pre-conditioning steps were done prior to connector assembly, in combination with a defined cutting of the sheath and the outer jacket. The FEP layer wasn't cut after the second pre-conditioning anymore.

The tensile strength of the extruded Liquid Crystal Polymer (LCP) fibers was found to be very high. Manual pull tests have been made with pliers pulling at both sides of 3-4 cm long samples - it was impossible to tear them apart. An observed disadvantage of this material is that it easily splits in longitudinal direction when the LCP tube is being compressed, especially if the LCP wall thickness is very thin and not mechanically supported underneath.

\subsection{Fiber connector}

The need for a fully non-magnetic optical connector design excluded taking available space qualified connector series (e.g., AVIM series by DIAMOND SA). Commonly used materials like austenitic steels are still too magnetic (remanent flux density is usually at least several $\mathrm{nT}$ at $2 \mathrm{~cm}$ distance) to be used in the vicinity of a high precision magnetometer sensor. Especially the spring, which keeps the ferrule with the optical fiber in place, is often problematic in terms of magnetic properties.

Schaefter+Kirchhoff had already successfully designed a practically non-magnetic FC/APC optical connector (flux density less than $50 \mathrm{pT}$ at $2 \mathrm{~cm}$ distance), its metal parts being made of titanium and aluminium only. This connector is used for the scalar magnetometer onboard the China Seismo-Electromagnetic Satellite (CSES) (in orbit since February 2018) [4][5].

The existing connector design had to be modified, taking into account the new fiber jacket (materials and setup) as well as the extended thermal range required for the JUICE mission.

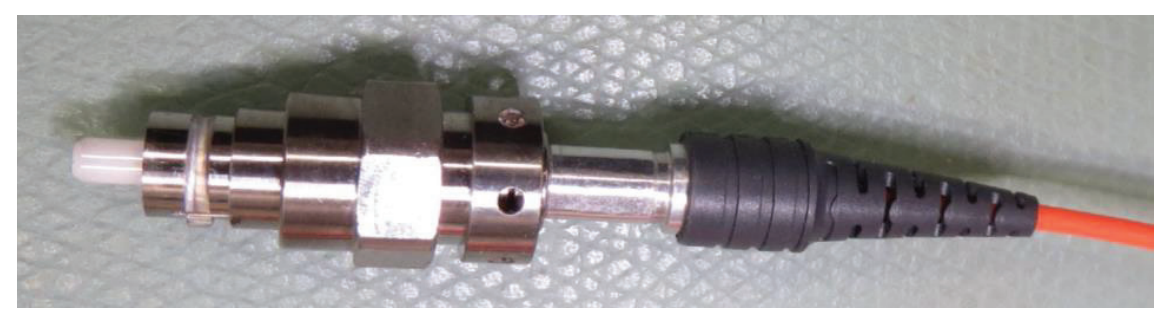

Figure 4. JUICE FC/APC connector by Schaefter+Kirchhoff. Clearly visible in the picture are the ferrule, the titaniumaluminium body, the kink protection and the orange FEP jacket of the fiber cable.

The plug design also includes an anti-twist feature. This is designed in such a way that longitudinal displacement is provided so that the different CTE's can be absorbed (see chapter 4). Two slightly different designs have been manufactured, one for the $50 \mu \mathrm{m}$ fiber cable assembly, one for the $400 \mu \mathrm{m}$ fiber cable assembly [10].

All connector parts were magnetically tested at IWF prior to assembly in order to verify the fully non-magnetic design.

Pure Zirconium oxide ferrules have been used as they could be procured with tighter tolerances to the diameter of the optical fibers (compared to ferrules with titanium inlays) resulting in less glue deposition between fiber and ferrule and better centricity of the fiber within the ferrule. The polyimide coating was removed from the fiber sections within the ferrule for the same reason. The polyimide removal was done by immersion into hot concentrated (96\%) sulfuric acid $\left(+180^{\circ} \mathrm{C}\right)$ at TUG in order to avoid mechanical stress onto the fiber underneath.

\subsection{Bending protection}

Hytrel 8086 sleeves produced by Diamond SA were used as bending protection for the interface between optical fiber cable and connector. Prior to assembly, the sleeves were vacuum baked for 24 hours at temperatures between $110^{\circ} \mathrm{C}$ and $125^{\circ} \mathrm{C}$ and $10^{-2}$ Torr to reduce outgassing. 


\subsection{Length of optical path and number of interconnects}

A total optical path length of around $20 \mathrm{~m}$ is needed to guide the light from the laser unit (located inside the JUICE satellite within a radiation protected vault) to the scalar magnetometer sensor at the tip of the $10.5 \mathrm{~m}$ long magnetometer boom, which will be deployed soon after the launch. The risk of reducing the mechanical and thermal stability of the optical path by splitting it into several separate fibers (adding additional optical connectors and interconnects) was weighed against the more complicated design of a non-separable interface between the boom and the satellite. In which, due to the greater fiber length, the strain movements that must be accommodated by the connectors are larger. To counteract this, only one optical interconnect - located at the boom root - per fiber path is used. The interconnect separates the optical path into a $13.84 \mathrm{~m}$ long fiber along the boom and a $6.14 \mathrm{~m}$ long fiber within the spacecraft. To be as close as possible to the final (flight) configuration, the optical fibers were thermally characterized as pairs in mated configuration. The optical interconnects were vibrated and shock tested to mission requirements in representative mounting configuration.

\section{FIBER ASSEMBLY TEST METHODS}

\subsection{Tests in thermal chamber}

A series of tests was done in thermal chambers to evaluate the shrinking behavior, the thermal expansion/contraction of the different jacket layers and the attenuation of optical power through the fiber due to thermal stress.

For the CTE test, a $15 \mathrm{~m}$ long, thermally preconditioned $400 \mu \mathrm{m}$ cable assembly ( 60 thermal cycles between $+140^{\circ} \mathrm{C}$ and $-30^{\circ} \mathrm{C}, 2^{\circ} \mathrm{C} / \mathrm{min}, 20$ minutes dwell time) without connectors was taken. The layers of the fiber jacket (LCP and FEP) and the optical fiber itself were glued together on the one end with epoxy glue. The other side of the fiber assembly remained loose. The fiber was coiled in a large radius (rectangular shaped, $\sim 50 \mathrm{~cm}$ wide and $\sim 80 \mathrm{~cm}$ high) and placed inside a climatic chamber at ambient pressure.

A graph paper (mm scale) was fixed with polyimide tape onto the FEP part of the "loose fiber end" (see Figure 5). The temperature was cycled between $+100^{\circ} \mathrm{C}$ and $-40^{\circ} \mathrm{C}$, with several hours dwell time at every temperature plateau.

At every temperature plateau, a photo was taken to record the distance between the optical fiber and the two jacket layers. The setup for this test is shown in Figure 5.
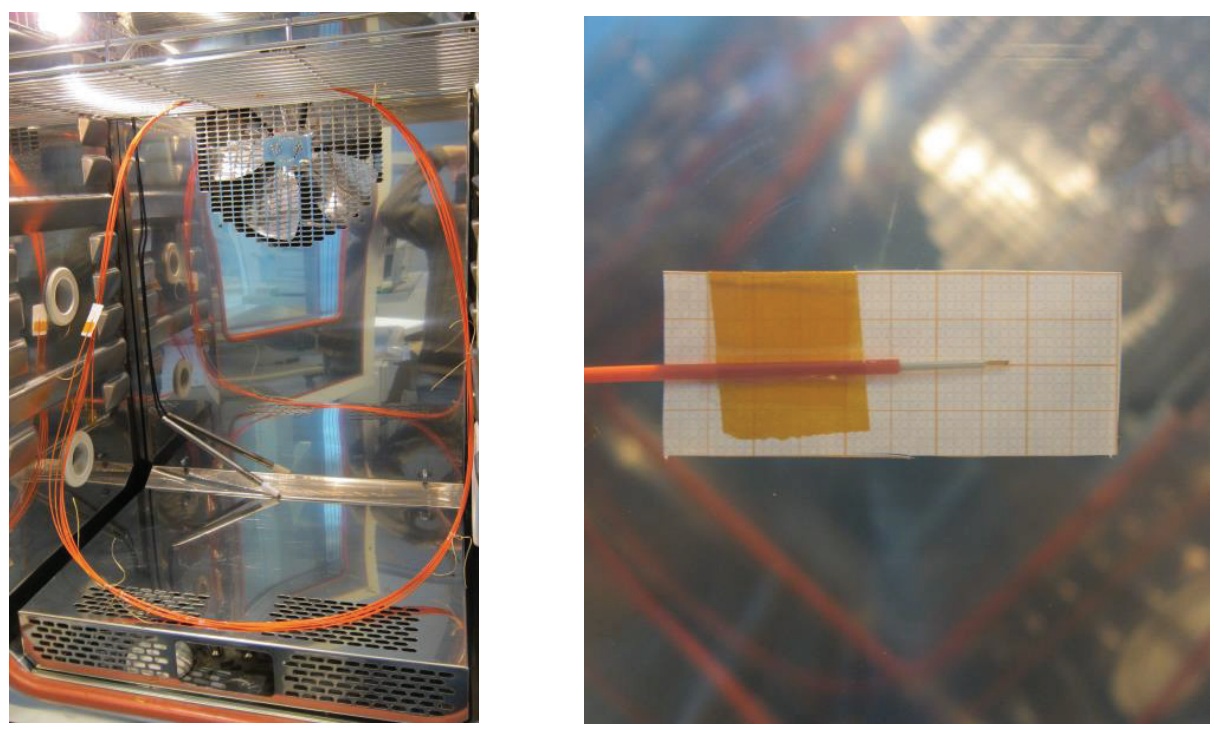

Figure 5. Coiled jacketed optical fiber without connectors mounted within the thermal chamber for a CTE test of the fiber jacket layers (left), mm scale fixed with Kapton tape to FEP layer of optical fiber jacket (right). Distance between fiber tip and fiber jacket layers was recorded at each temperature plateau $\left(+100^{\circ} \mathrm{C}\right.$ and $\left.-40^{\circ} \mathrm{C}\right)$ for 19 thermal cycles. 
The measurements confirmed the good thermal matching of the CTEs of the LCP sheath and the optical fiber. The distance between LCP sheath and the fiber tip got reduced by $\sim 0.3 \mathrm{~mm}(0.02 \mathrm{~mm}$ per $\mathrm{m}$ fiber length) after the first cooling cycle but didn't change visibly for the rest of the cycles anymore. On the other hand, the FEP jacket layer showed further shrinking as well as thermal expansion and contraction with respect to the optical fiber. The two effects are well separable from each other with this method (see Figure 6). Whereas the CTE mismatch between FEP and the glass fiber resulted in a recurring length difference of about $1 \mathrm{~mm}$ between the two temperature extremes, the shrinking of the FEP has a saturation like behavior, getting less with the increasing number of thermal cycles.

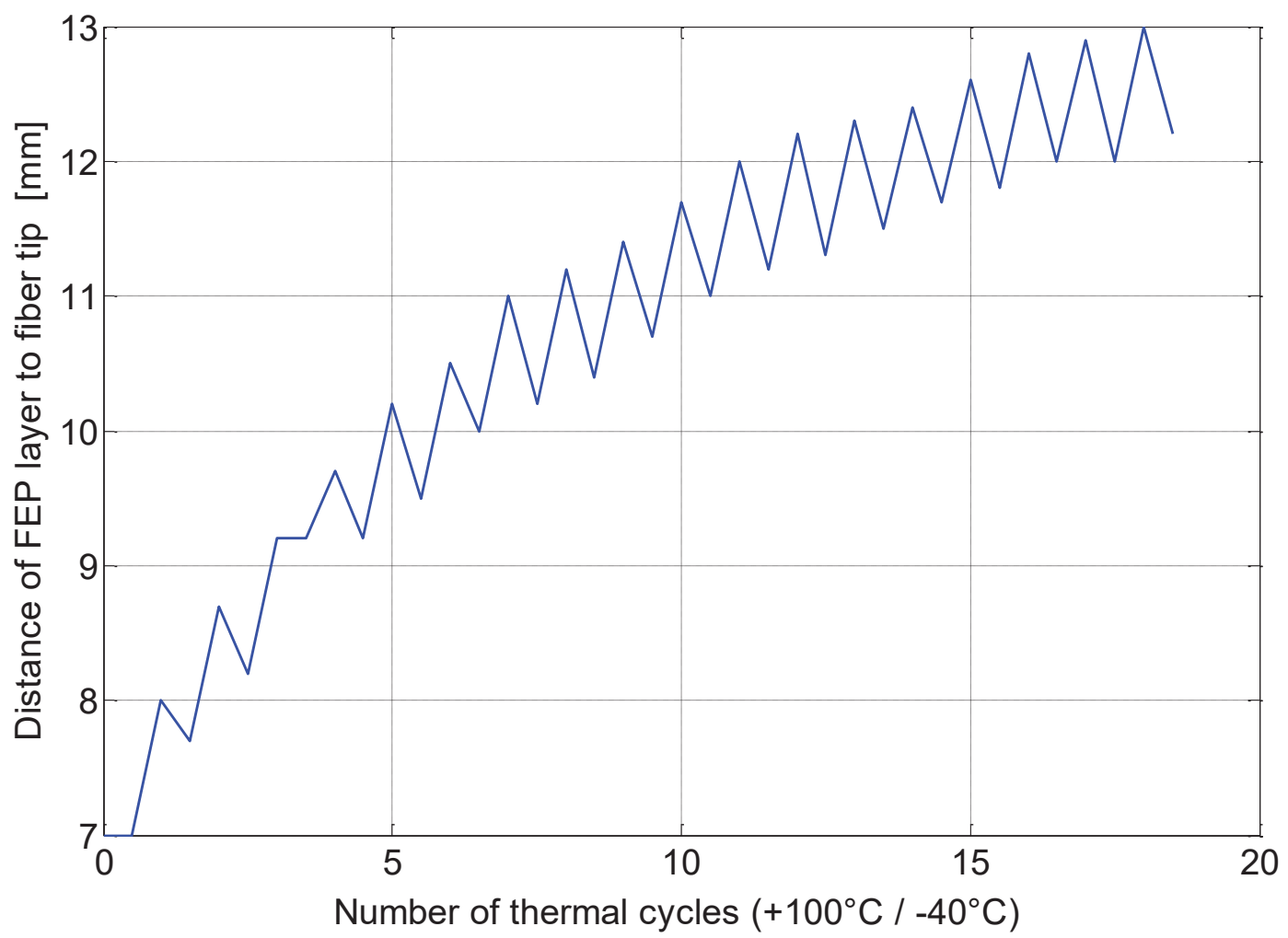

Figure 6. Shrinking and thermal expansion/contraction of FEP fiber jacket layer with respect to the optical fiber as a function of thermal cycles

Another set of thermal test series was done later with the connectorized fiber cable assemblies. In each test, two fibers have been thermally cycled together. Except for approximately $1 \mathrm{~m}$ of fiber length at each end, the fiber assemblies were placed within a thermal chamber. The test setup is shown in Figure 7. 


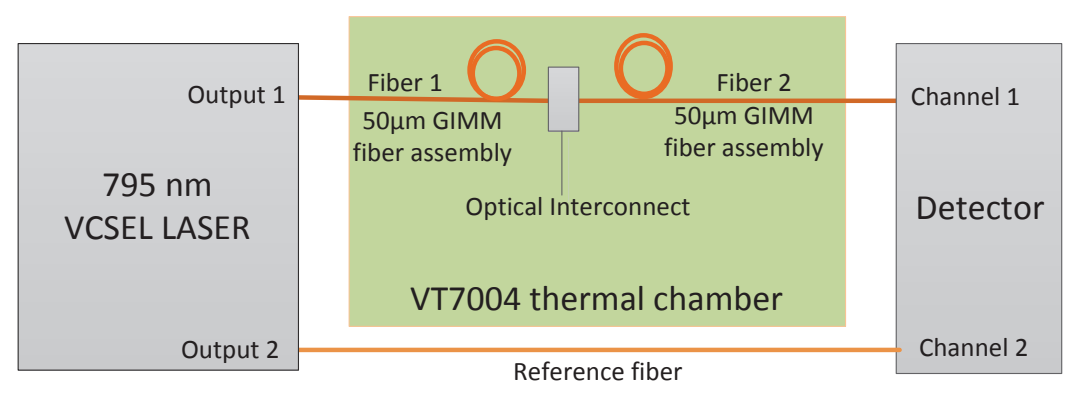

Figure 7. Schematic setup of thermal cycling tests of the connectorized fiber cable assemblies with monitoring of optical power. A reference path is used to compensate possible power variations of the VCSEL laser source.

The optical power through the complete optical path was monitored during the thermal cycling. Figure 8 shows the measured optical power during a thermal cycling test with a mated pair of JUICE $50 \mu \mathrm{m}$ pre-test fiber assemblies. In the test scenario, a $13.7 \mathrm{~m}$ long fiber was mated to a $19.7 \mathrm{~m}$ long fiber, the total length of the optical path being $33.4 \mathrm{~m}$.

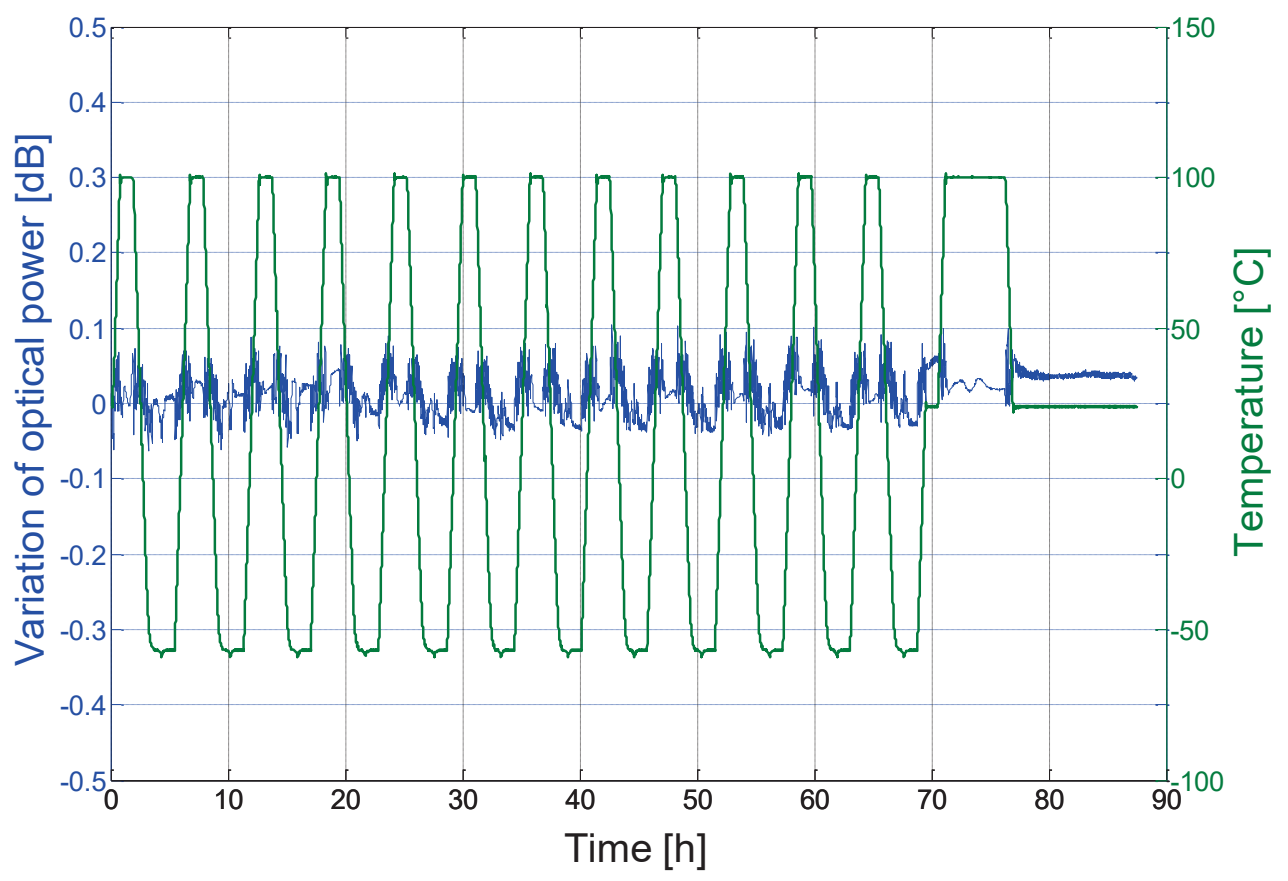

Figure 8. Variation of optical power with thermal cycling of a mated pair of JUICE $50 \mu \mathrm{m}$ optical fiber assemblies (pre-test for final fiber production, $13.7 \mathrm{~m}$ long fiber mated with $19.7 \mathrm{~m}$ long fiber, total length of tested optical path: $33.4 \mathrm{~m}$ ). Therefore, the length normalized variation with temperature is around $0.003 \mathrm{~dB} / \mathrm{m}$. 


\subsection{X-ray inspection}

X-ray examination of optical fibers turned out to be the method for detecting deficiencies in the optical path. It was kept later on as an acceptance test for the flight fiber cable assemblies. The connectors have been X-rayed for product assurance of their manufacturing (e.g., presence of metal cuttings) as well as to determine the position of the fiber and the fiber jacket layers after the full set of screening tests including thermal cycling. Figure 9 shows a JUICE FC/APC optical fiber connector during X-ray inspection. On the X-ray image, the optical fiber is visible as a light grey shadow in the center of the fiber connector. This image shows a problem that occurred in the early design phase of the $50 \mu \mathrm{m}$ optical fiber assembly. During cooling to $-170^{\circ} \mathrm{C}$, the fiber assembly suddenly showed an increased attenuation of optical power which didn't disappear after warming up. The source of this attenuation, a bending of the fiber within the connector, was found with the help of an X-ray inspection. The source of the problem - insufficient stress relief between optical fiber and fiber jacket layers - was resolved with design changes in both, the fiber jacket and the connector, thereafter.
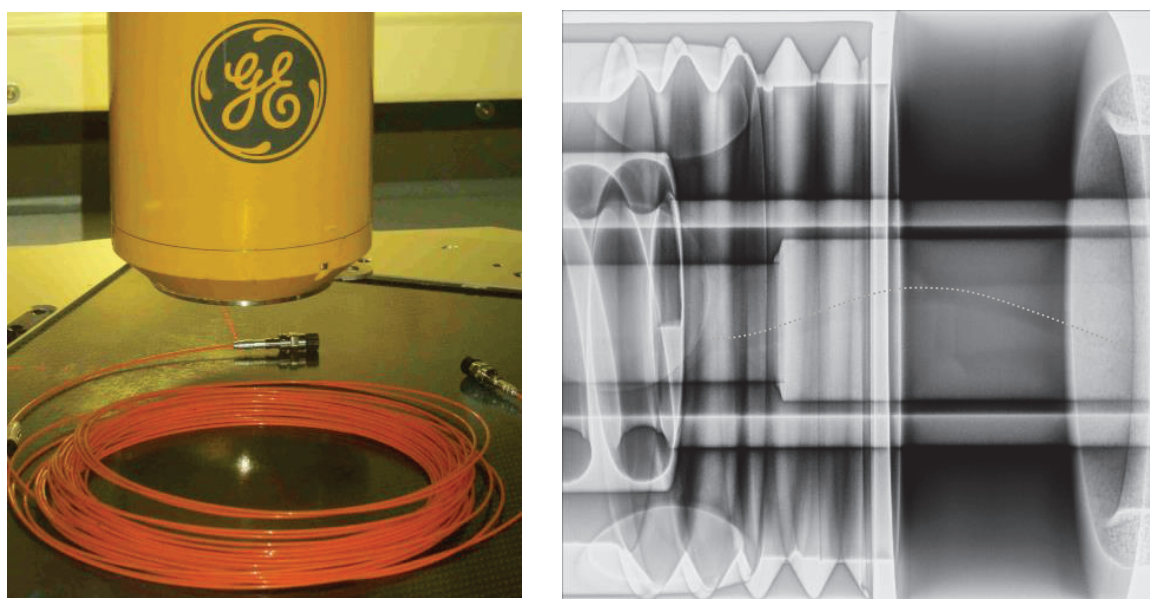

Figure 9. Test setup for X-ray inspection of optical fiber connectors (left) and X-ray image detail showing a bent optical fiber (marked as dotted line) within a FC/APC optical fiber connector (right). This was found to be the cause for a highly increased attenuation of optical power through the fiber after cooling to $-170^{\circ} \mathrm{C}$.

\subsection{Light scattering tests}

Several tests with powerful laser sources have been done in order to find major and minor defects within the fiber assemblies. Where the optical fiber is mechanically stressed (e.g., bent) within the fiber jacket, light will escape the fiber core and the cladding. If the laser source is powerful enough and the wavelength is selected in a way that the light can protrude the polymeric materials of the coating and the fiber jacket layers, then these locations become visible from outside as bright spots or regions.

Two different laser sources were used to test the JUICE fiber assemblies: An infrared laser (795 nm, $25 \mathrm{~mW})$ as well as a visible helium neon laser $(633 \mathrm{~nm}, 700 \mu \mathrm{W})$.

For all tests with fibers with assembled FC/APC connectors, laser light was coupled into a pre-fiber with a laser beam coupler. The pre-fiber was connected with an optical interconnect to the fiber under test (Setup 1 of Figure 10).

For all tests with non-connectorized fiber assemblies (jacketed fibers without connectors), the fiber under test was directly connected to the laser beam coupler with a bare fiber adapter supplied by Schaefter+Kirchhoff (Setup 2 of Figure 10). The advantage of this setup was that mechanical stress onto the fiber inside the fiber jacket (after fiber insertion and thermal preconditioning) could be detected prior to connector assembly. 
In both setups, the fiber under test was wound onto a cylindrical tube of about $11 \mathrm{~cm}$ diameter. The transmitted optical power through the fiber was monitored with an optical power meter (see Figure 10).
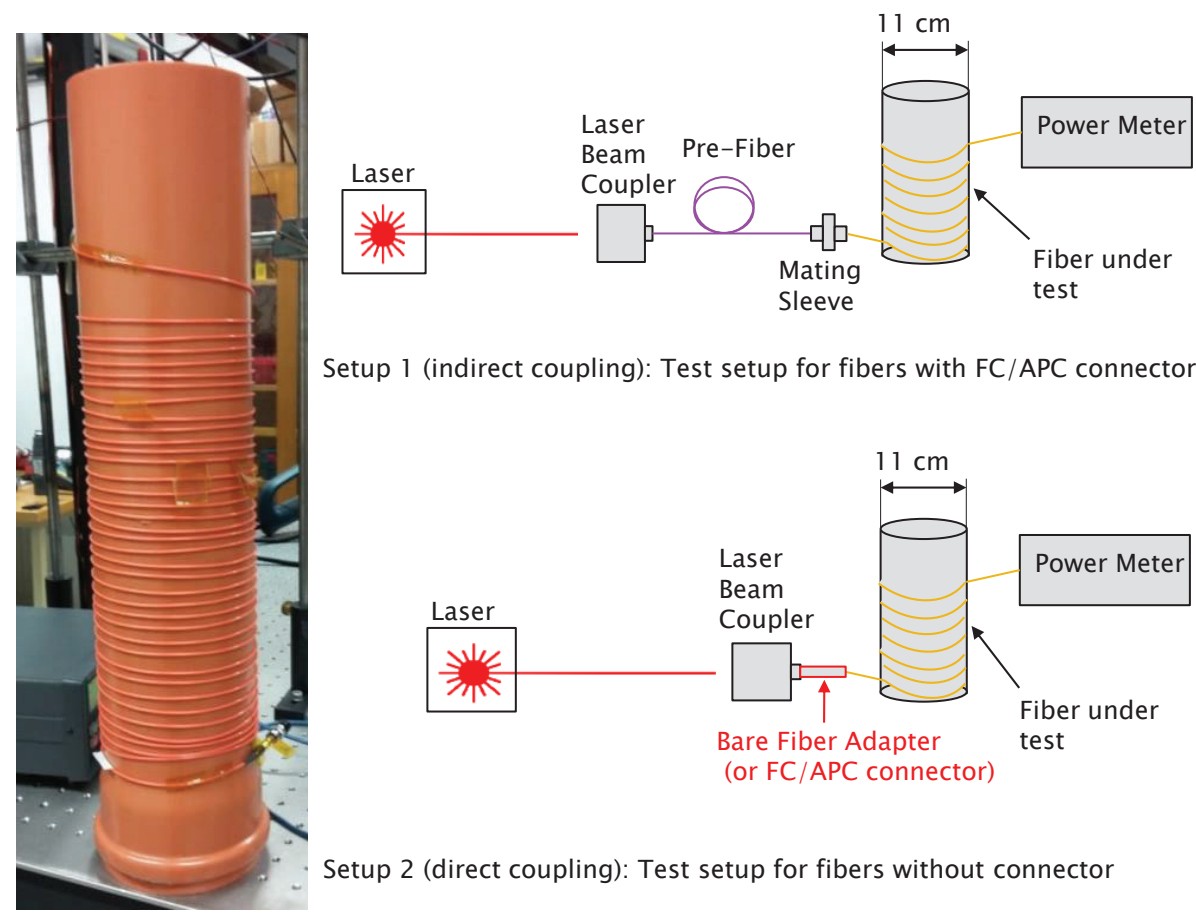

Setup 1 (indirect coupling): Test setup for fibers with FC/APC connector

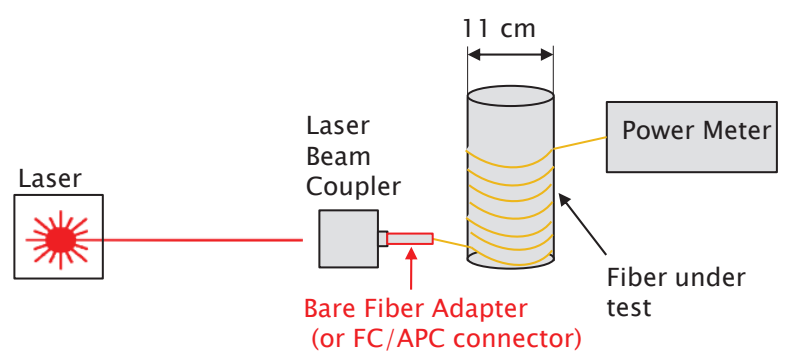

Setup 2 (direct coupling): Test setup for fibers without connector

Figure 10. Photo and schematics of test setup for laser light inspection with use of a He-Ne-Laser with a wavelength of $632.8 \mathrm{~nm}$. A photo (right) taken of the fiber under test shows clearly the areas of mechanical stress.

The positions of the bright locations found with the help of this visible laser light inspection (see Figure 10, right image) were marked and additionally inspected in detail with X-rays. The source of the bright regions was found to be scrambled strands of the LCP layer that caused micro-bendings of the optical fiber. The problem was resolved by increasing the wall thickness of the LCP sheath to improve its lateral strength. 


\subsection{Low temperature tests}

Due to the harsh requirements of the JUICE mission, the optical fiber assemblies had to be tested down to temperatures between $-160^{\circ} \mathrm{C}$ and $-190^{\circ} \mathrm{C}$ (see Table 1). A dedicated setup was developed at TUG (which is shown in Figure 11). For the test the coiled fibers are placed on a special aluminum holder. This holder is lowered into a big Dewar partially filled with liquid nitrogen. The opening of the Dewar was sealed with thermally insulating material. The setup includes a little fan, which is also mounted onto the holder. When the fan is turned on, the circulating cold nitrogen gas cools the aluminum holder and the fiber assembly within. During the test, the temperature is permanently measured with a Pt1000 sensor which is loosely placed within the coiled fiber. The optical power through the fiber assembly is monitored throughout the test.
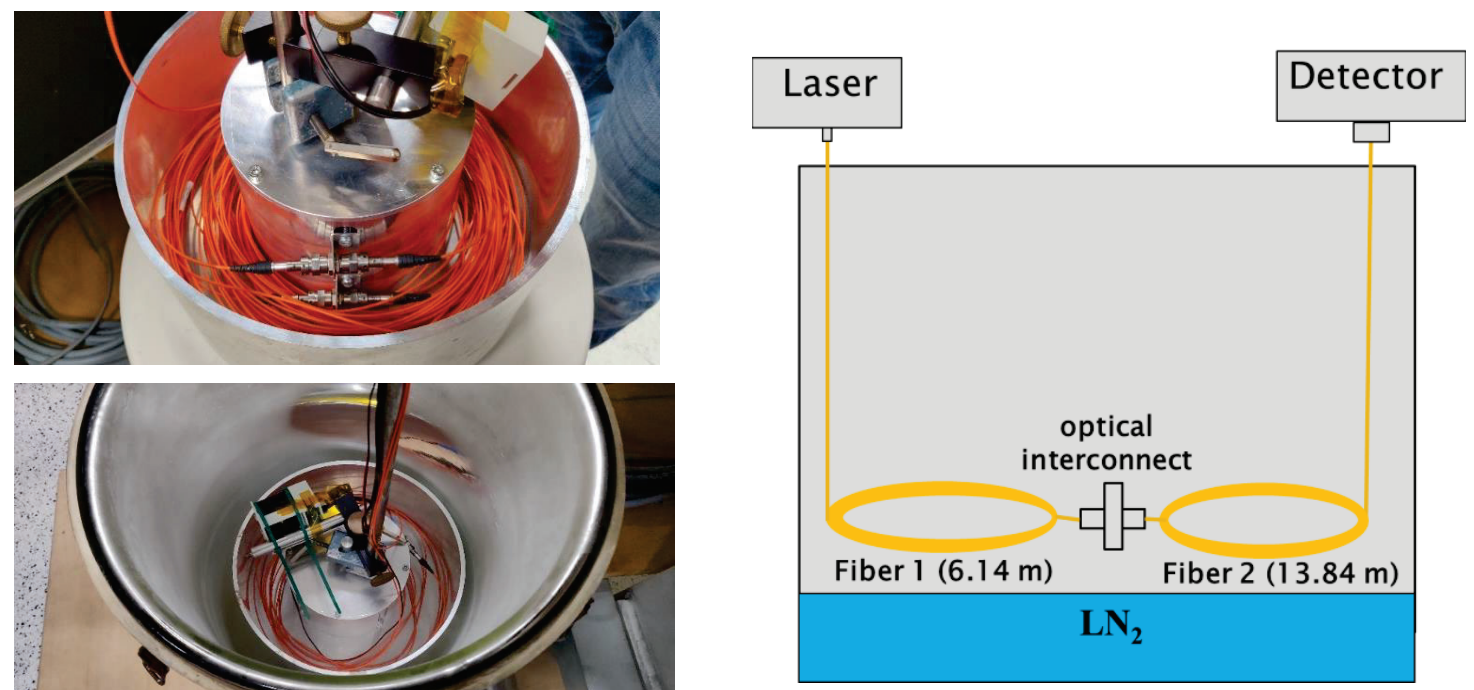

Figure 11. Photos and schematic of typical test setup for low temperature tests

Test temperatures to about $-175^{\circ} \mathrm{C}$ have been achieved with this setup. The optical fiber assemblies were tested standalone as well as in mated conditions (in pairs or triplets) with the optical interconnects being cooled down to the same low temperature regime. Figure 12 shows the variation of optical power through a mated pair of $50 \mu \mathrm{m}$ fibers during a low temperature test. The total length of the optical path including one optical interconnect was nearly $20 \mathrm{~m}$ which was realized with a $6.14 \mathrm{~m}$ long fiber being mated to a $13.84 \mathrm{~m}$ long fiber.

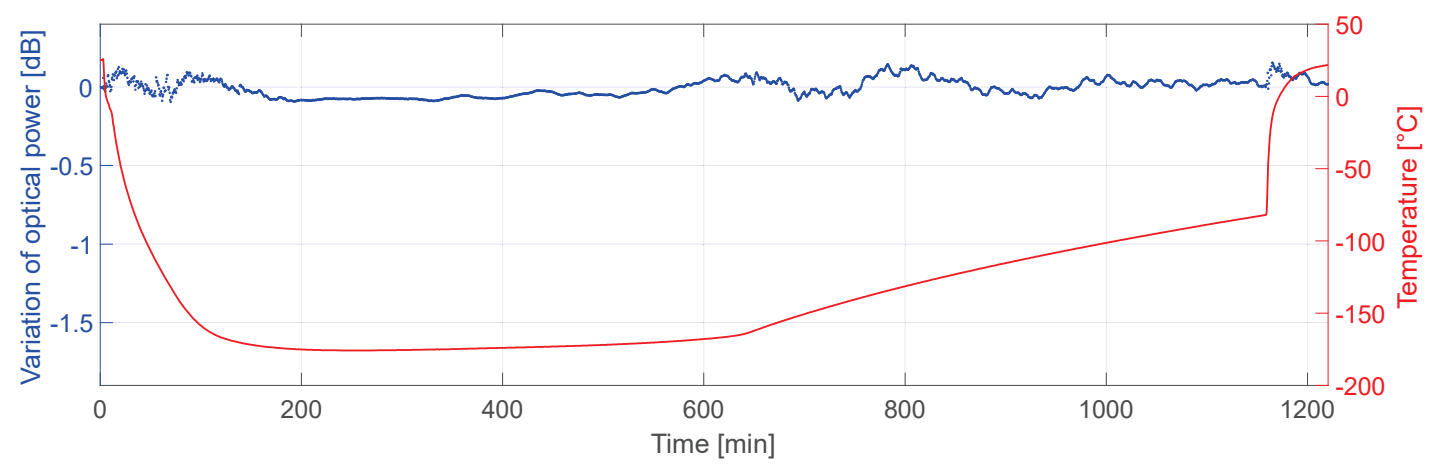

Figure 12. Low temperature test with monitoring of optical power through a tested pair of $50 \mu \mathrm{m}$ multimode optical fiber assemblies. The total length of optical path including one optical interconnect was $20 \mathrm{~m}$. 


\section{SCREENING TESTS}

With the experience of the pre-tests done during the development of the optical fiber cable assembly, a full set of screening tests was defined by IWF in order to verify the reliability of the manufactured optical fiber cable assembly [11]. The screening of the flight fiber assemblies included the following tests:

- Visual inspection of the fiber assembly

- End face visual inspection at the beginning and at the end of the screening tests

- Magnetic cleanliness check in a triple $\mu$-metal shield

- Mechanical fit check of connectors

- High temperature stress test including connectors $\left(5\right.$ hours at $\left.+100^{\circ} \mathrm{C}\right)$

- High temperature stress test without connectors $\left(5\right.$ hours at $\left.+120^{\circ} \mathrm{C}\right)$

- $\quad 12$ thermal cycles $+100^{\circ} \mathrm{C} /-60^{\circ} \mathrm{C}$ in mated configuration with monitoring of the optical power

- Low temperature stress test including connectors (min. 5 hours at $-170^{\circ} \mathrm{C}$ )

- 1-2 low temperature tests in mated configuration with monitoring of the optical power $\left(\geq 5\right.$ hours at $\left.170^{\circ} \mathrm{C}\right)$

- Full performance check of the mated fibers in magnetometer measurement configuration

- X-ray inspection.

\section{QUALIFICATION TEST CAMPAIGN}

\subsection{Lot acceptance test of optical fiber assemblies}

In order to finally qualify the design of the optical fiber assemblies for the requirements of the Jupiter mission, a lot acceptance test program was defined [11] and successfully carried out. A total of 6 fiber assemblies per fiber type (12 fibers assemblies in total) were subject to tests listed in Table 2.

Table 2. Lot acceptance test program of the optical fiber assemblies for the JUICE J-MAG scalar magnetometer

\begin{tabular}{|c|c|}
\hline \multicolumn{2}{|l|}{ Mechanical Tests } \\
\hline Torsion test & $3 \mathrm{~N}$ tensile load, twist angle ( 1 cycle $): \pm 180^{\circ}$, number of twists: 25 \\
\hline Static side load test & $1 \mathrm{~N}$ for $10 \mathrm{~min}$ at $90^{\circ}$ to the connector longitudinal axis \\
\hline Retention test & $5 \mathrm{~N}$ for $2 \mathrm{~min}$ along the connector longitudinal axis \\
\hline \multicolumn{2}{|l|}{ Environmental Tests } \\
\hline Vibration & Random vibration up to $17.3 \mathrm{~g}_{\mathrm{rms}}$ with mated fiber assemblies \\
\hline Shock & $\begin{array}{l}2 \text { axes (longitudinal axis and perpendicular axis) with mated fiber assemblies } \\
3 \text { shocks per axis, } 100 \mathrm{~Hz}: 20 \mathrm{~g} ; 1000 \mathrm{~Hz}: 700 \mathrm{~g} ; 10000 \mathrm{~Hz}: 700 \mathrm{~g}\end{array}$ \\
\hline High temperature test & Accelerated test, 380 hours at $+140^{\circ} \mathrm{C}$ \\
\hline Thermal cycling test & $\begin{array}{l}50 \text { cycles, } 3 \text { different temperature zones realized within one test, fibers tested in } \\
\text { triplets, using } 2 \text { optical interconnects per fiber path, monitoring of optical power } \\
-190^{\circ} \mathrm{C} /+120^{\circ} \mathrm{C} \text { (qualification range for the opt. fiber assembly along the boom) } \\
-160^{\circ} \mathrm{C} /+100^{\circ} \mathrm{C} \text { (qualification range for optical fiber connectors) } \\
-80^{\circ} \mathrm{C} / 45^{\circ} \mathrm{C} \text { (qualification range for optical interconnects) }\end{array}$ \\
\hline
\end{tabular}

The thermal cycling tests have been conducted by Alter Technology TÜV Nord S.A.U. using three thermal chambers in parallel to realize the three different climatic zones for the optical paths being tested [12]. 


\subsection{Low temperature irradiation tests of bare fiber}

The low temperature irradiation tests were not part of the lot acceptance test program for the jacketed and connectorized fiber cable assemblies. These tests could only be performed on the bare fiber from the same fiber lot, due to the limited space within the cryostat and the feedthrough possibilities. Nevertheless, these tests were essential for the fiber selection, as the radiation induced attenuation at low temperatures is expected to be the main source of fiber degradation during the JUICE space mission. Based on a refined radiation model by ADS experts and additional modelling of details by University of Leicester, four irradiation test campaigns were conducted by Fraunhofer INT. Fibers of five different manufactures were tested at dose rates of $150,3.4$ and $0.3 \mathrm{rad} / \mathrm{s}$, at temperatures of $40 \mathrm{~K}$ and $100 \mathrm{~K}$ and up to a maximum TID of $16 \mathrm{Mrad}$ [13]. The finally selected fiber types from Fibercore and Molex/Polymicro were found to be compliant with the available optical budget in terms of radiation induced attenuation.

\section{CONCLUSION}

Two different types of non-magnetic fiber assemblies, a $50 \mu \mathrm{m}$ gradient index multimode fiber assembly and a $400 \mu \mathrm{m}$ step index multimode fiber assembly have been developed and successfully qualified to the harsh environmental requirements of the European space science mission to the icy moons of Jupiter (JUICE).

\section{ACKNOWLEDGEMENTS}

Thanks to everybody that contributed to this fiber optic assembly development and qualification program. A joint effort of all involved parties (ESA, research institutions and industry) was vital to achieve the required performance. Apart from the uncountable hours spent by some members of the J-MAG scalar magnetometer instrument team in Graz throughout the design, manufacturing and test phases, a lot of experts at different research institutions were needed (and willing) to share their knowledge for this development. All industry partners showed incredible patience (being faced with a never-ending list of requirements, non-conformances and due dates) and contributed design ideas based on knowledge in their own special fields. Thanks to AIRBUS D\&S, who has had the lead responsibility for this fiber optic assembly development, for being the driving force behind this program and for the big add-on support in many fields. Thanks also to ESA for guidance and expertise throughout the whole process, without these valuable inputs we wouldn't finally have succeeded.

I'd like to direct my special thanks to Melanie Ott from NASA Goddard Space Flight Center. Many design decisions have been taken based on the extensive work of her team and their publications in this area [14-22].

\section{REFERENCES}

[1] AIRBUS D\&S, "MagSCA Fibreoptics Design Description”, JUI-ADSF-MAG-DD-000121, iss.1, JUICE project technical document (2019)

[2] Jernej, I., "J-MAG MAGSCA Specification of Optical Fiber Jacket", JMAG-SCA-TN-005, JUICE project technical document, iss. 1 (2017)

[3] Kuhnhenn, J., "Radiation Issues in Fibers", ICAN Workshop Jena (2012)

[4] Pollinger, A., et al.," Coupled dark state magnetometer for the China Seismo-Electromagnetic Satellite", Meas. Sci. Technol. 29095103 (2018)

[5] Pollinger, A., et al.," In-orbit results of the Coupled Dark State Magnetometer aboard the China SeismoElectromagnetic Satellite", Geoscientific Instrumentation Methods and Data Systems 9(2), 275-291 (2020)

[6] W.L. Gore \& Associates GmbH , "GSC-13-84815-00 Fiber Optic Multimode step-index Cable”, data sheet (2015)

[7] Heraeus Fused Silica Knowledge Base, "Properties of fused silica", Heraeus Conamic, 2021, https://www.heraeus.com/en/hca/fused silica quartz knowledge base 1/properties 1/properties hca.html\#tabs -608478-5 (3 February 2021)

[8] O'Riorden, S., Mahapatra, A., "Radhard optical patchcords and packaging for satellites using liquid crystal polymers", Proc. of SPIE 10564, 105641I-1 - 105641I-11 (2012) 


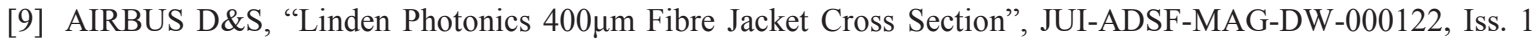
(2018)

[10] AIRBUS D\&S, "Schäfter\&Kirchhoff Drawing FC/APC Connector $400 \mu \mathrm{m}$ and $50 \mu \mathrm{m}$ Opt. 1b", JUI-ADSFMAG-DW-000123, Iss. 1 (2019)

[11] AIRBUS D\&S, “JUICE Optical Harnesses LAT plan”, JUI-ADST-SYS-PL-000168, Iss. 4 (2019)

[12] Barbero, J., Jernej, I., "Monitoring of Optical Fibers during cycles keeping the optical connectors at a different stable temperatures", Iss. 4 (2020)

[13] Kuhnhenn, J., Weinand, U., Wolf, R., „Co-60 gamma irradiation of several fibres at cryogenic temperatures for JUICE”, JUI-ADSF-MAG-TR-000121, Iss. 1.0, Fraunhofer INT (2017)

[14] Ott, M., et al., NASA Electronic Parts and Packaging Radiation Effects Summary Database on Commercially Available Optical Fiber, NASA GSFC (2009)

[15] Ott, M., Plante, J.,Shaw, J., Garrison Darrin, M.A., "Fiber Optic Cable Assemblies for Space Flight: Issues and Remedies,", Paper number 975592 AIAA/SAE World Aviation Congress, Anaheim, CA, pp. 1-7 (1997)

[16] Ott, M., "Fiber optic cable assemblies for space flight applications II:thermal and radiation effects", NASA GSFC (1997)

[17] Ott, M., "Fiber optic cable assemblies for space flight applications III: Characterization of Commercial Cables for Thermal Effects", NASA GSFC (1999)

[18] Ott, M., et al., "Optical fiber cable assembly characterization for the Mercury Laser Altimeter", Proc. of SPIE 5140, Enabling Photonic Technologies for Aerospace Applications V (2003)

[19] Ott, M., Friedberg, P.," Technology validation of optical fiber cables for space flight environments", Proc. of SPIE 4216, Optical Devices for Fiber Communication II (2001)

[20] Thomes, W. J., et al, “Fiber optic cable thermal preparation to ensure stable operation”, Proc. of SPIE 7070, Optical Technologies for Arming, Safing, Fuzing, and Firing IV, 70700B (3 September 2008)

[21] Lindensmith, C. A., et al., "Development and qualification of a fiber optic cable for martian environments", Proc. of SPIE 10565, International Conference on Space Optics - ICSO 2010, 1056519 (2017)

[22] Thomes, W.J., et al., "Cryogenic fiber optic assemblies for spaceflight environments: design, manufacturing, testing, and integration", Proc. of SPIE 9981, Planetary Defense and Space Environment Applications, 99810F (2016)

[23] Berghmans F., Brichard B., Fernandez A.F., Gusarov A., Uffelen M.V., Girard S. (2008) An Introduction to Radiation Effects on Optical Components and Fiber Optic Sensors. In: Bock W.J., Gannot I., Tanev S. (eds) Optical Waveguide Sensing and Imaging. NATO Science for Peace and Security Series. Springer, Dordrecht. https://doi.org/10.1007/978-1-4020-6952-9_6 